\title{
Criterios \\ Técnicas no destructivas aplicadas a la conservación del patrimonio arquitectónico. Colorimetría
}

\author{
Giuseppe Cultrone' $>$ Olga Cazalla' $>$ Carlos Rodriguez' $>$ Ma José de la Torre" $>$ Eduardo Sebastián'
}

\section{Resumen}

El artículo presenta un estudio en el que se ha medido mediante colorimetria la modificación de luminosidad y cromaticidad de ladrillos macizos cocidos a distintas temperaturas. Tierras arcillosas de diferente composición generan piezas de diferentes colores porque reflejan el desarrollo de distintas fases minerales. Se ha demostrado también la utilidad de la colorimetría en la elección de la cantidad más idónea de aditivo a añadir a la materia prima arcillosa sin que se verifiquen cambios sustanciales en el color de las piezas cerámicas.

\section{Palabras clave}

\section{Técnica no destructiva}

\section{Colorimetría}

Conservación

Patrimonio arquitectónico

Ladrillo

Mineralogia

Aditivos

\section{Introducción}

El uso de la colorimetría tiene una aplicación relevante en el campo de la conservación del Patrimonio Arquitectónico. La variación de determinados parámetros relacionados con el color de un material indica una modificación estética más o menos importante.

El color de los materiales pétreos depende de las fases minerales presentes y de la textura. Los minerales, aunque estén en baja proporción, son responsables de la pigmentación de los materiales. También el tamaño de las partículas y su disposición puede influir en el color y brillo del material, siendo las rocas de grano fino las que tienen colores más homogéneos (Esbert et al., 1997).

El color es un término general que se refiere a la composición de la longitud de onda de la luz, y especialmente a su aspecto visible. Se describe a la luz como formada por un determinado color cuando prevalece la energía de una o más regiones dentro de la gama de longitudes de onda que van desde 380 a 720 nm (espectro visible). La predominancia de una región u otra en la composición espectral suele manifestarse ante el observador como diferencias de tonalidad o saturación. Por lo general, el color se presenta al individuo mediante la reflexión en las superficies de objetos sobre los cuales incide la luz que puede considerarse blanca. Así, una superficie roja es aquélla que absorbe la mayor parte de la luz de onda corta y refleja la luz de longitud de onda larga hacia el ojo. En función de su longitud de onda se va a estimular de forma diferente la retina humana y establecer el espectro de color, disponiéndose en orden el rojo, naranja, amarillo, verde, azul y violeta. De estos colores, el rojo, el verde y el azul son considerados como colores primarios. A partir de la mezcla de ellos se obtienen todos los demás.

El sistema de representación de los colores más utilizado es el de Munsell (1977), que es bastante sencillo pero está lejos de ser exacto ya que no evita el problema de posibles defectos de visión del observador, no tiene presente muchas veces el tipo de iluminante y produce terminologías confusas (se aplican diferentes nombres a colores iguales). Por ese motivo, se ha desarrollado y perfeccionado una ciencia sobre la identificación y la especificación de los colores: la colorimetría.

La colorimetría se ocupa de los métodos empleados para medir y expresar cuantitativamente el color en función de la cantidad de luz reflejada por una superficie analizada. La colorimetría surge con la teoría tricromática de la visión de los colores, la cual establece que pueden elegirse tres colores primarios de forma tal que, combinados en diversas proporciones, puedan igualar a cualquier color desconocido. Por lo tanto, la colorimetría consiste en la designación de los tres primarios y en la determinación de la cantidad de cada uno de ellos que debe emplearse para reproducir el color requerido. 
Los colores se especifican a menudo en un diagrama bidimensional, llamado diagrama de cromaticidad y recomendado por la CIE (Commission Internationale de l'Eclairage), el cual muestra las relaciones entre los valores triestimulares independientemente de la luminosidad. En el sistema de color CIELab, introducido en el año 1976, distancias iguales son aproximadamente diferencias iguales en la percepción del color. Los valores $L^{*} a^{*} b^{*}$ se definen a partir de los valores triestímulo de un objeto (Wyszecki y Stiles, 1982).

El sistema está basado en la teoría de colores opuestos: un color no puede ser al mismo tiempo amarillo y azul o rojo y verde. Por esta razón estos colores se sitúan en los extremos del diagrama de medidas del color.

$L^{*}$ es la variable luminosidad con valores comprendidos entre 100 (negro) y +100 (blanco), $a^{*}$ y b* son las coordenadas de cromaticidad, variando $a^{*}$ de -60 (verde) $a+60$ (rojo) y $b^{\star}$ de -60 (azul) a +60 (amarillo).

El sistema CIELCH utiliza el mismo diagrama que el anterior, pero trabaja con coordenadas cilindricas en vez de cartesianas. $L^{*}$ es la variable luminosidad, $\mathrm{C}^{*}$ es el color y $\mathrm{H}^{\circ}$ es el ángulo del matiz.

Muchas de las alteraciones cromáticas de los monumentos dependen de fenómenos de disolución y neoformación de fases mineralógicas debido a su exposición a la intemperie, en particular a la acción del agua (Cultrone et al., 2002), así como a la formación de pátinas o costras negras debido a contaminación atmosférica (Derbez y Lefevre, 1996), y de pátinas biológicas por la presencia de microorganismos (Paleni y Curri, 1976; Warscheid y Braams, 2000). Teniendo en cuenta estos hechos, el color podría ayudar a medir el grado de alteración alcanzado por los materiales de construcción o, también, el grado de contaminación del ambiente (Esbert et al., 1997). La contaminación se encuentra bastante restringida a ambientes urbanos, donde se localizan la mayoría de los edificios históricos actualmente existentes. En los últimos decenios, la acentuación del deterioro de los monumentos de las ciudades industrializadas ha sido atribuida al fuerte incremento de contaminantes atmosféricos (Rodriguez Navarro y Sebastián Pardo, 1994 y 1996). Uno de los efectos más indeseables es la disolución de $\mathrm{SO}_{2}$, procedente de los residuos de la combustión de gasoil y gasolina, en agua de humedad o de lluvia, presente en la superficie de los monumentos (Fassina, 1976).

Las variaciones del color pueden depender también de la presencia de otros materiales como estucos o morteros, de la reposición o sustitución de material, aunque proceda de la misma cantera, o bien por la utilización de productos consolidantes y/o hidrofugantes en intervenciones de conservación (Carreta y Giovannone, 1989). En la mayoría de los casos, en el momento de la aplicación de estos productos, no se aprecian variaciones en el color del material de construcción tratado, pero con el tiempo, dependiendo de la composición del producto de tratamiento así como de la exposición a las radiaciones solares, puede que el material modifique apreciablemente su cromaticidad y su luminosidad (García Pascua et al., 1988 y 1999; Durán Suárez, 1996). Debido a todas estas causas, son recomendables estudios previos sobre la variación del color en materiales que se verán sometidos a tratamientos de protección y consolidación.

Otra utilidad del procedimiento, en relación con la sustitución o reposición de material pétreo, es la posibilidad de agotamiento o inaccesibilidad de la cantera histórica, con lo que se hace necesario valorar el color de otras piedras similares procedentes de otros distritos canterables y establecer una escala que permita la elección del material más acorde cromáticamente con el del monumento en proceso de restauración (Sebastián y Zezza, 1998).

\section{Proceso experimental con ladrillos}

Se han estudiado las modificaciones cromáticas que sufren los ladrillos macizos (norma NBE-FL-90) de diferente composición tras la cocción. Las materias primas para la elaboración de los ladrillos proceden de dos localidades cercanas a la ciudad de Granada: Viznar y Guadix. Estas tierras arcillosas estaban ya seleccionadas y preparadas por la fábrica "Cerámicas San Francisco" (Granada) antes de que se mezclaran y moldearan a mano en laboratorio siguiendo el método artesanal. Mediante estas arcillas se han fabricado ladrillos cocidos a 700, 800, 900,1000 y $1100^{\circ} \mathrm{C}$, utilizando un horno eléctrico (Herotec CR35) con fuente de calor fija.

Mineralógicamente, el material de Viznar está compuesto esencialmente por cuarzo (40\%) y filosilicatos (30\%) y cantidades menores de calcita (15\%), dolomita (5\%) y feldespatos (10\%). En el de Guadix el cuarzo constituye la fase mineral más común (50\%); filosilicatos (40\%) y feldespatos (10\%) son los otros componentes. El análisis de la fracciónx $2 \mu \mathrm{m}$ no ha evidenciado diferencias sustanciales entre las dos materias primas. Ambas tierras están compuestas por ilita y cantidades menores de caolinita, clorita, esmectita y paragonita (Cultrone, 2001).

Las transformaciones de alta temperatura en los ladrillos de Guadix incluyen la formación de mullita más sanidina a costa de la moscovita y/o ilita, a temperaturas iguales o superiores a $800^{\circ} \mathrm{C}$. La mullita se forma por remplazamiento epitáctico de la moscovita (Rodríguez Navarro et al., 2003). En el caso de ladrillos de Viznar, la descomposición de los carbonatos favorece la formación de silicatos de $\mathrm{Ca}$ (y Mg) como gehlenita, wollastonita, diópsido y anortita. Mayores detalles sobre la mineralogía de los dos grupos de ladrillos y los procesos de transformación de las fases minerales están descritos en Cultrone et al., 2001. 


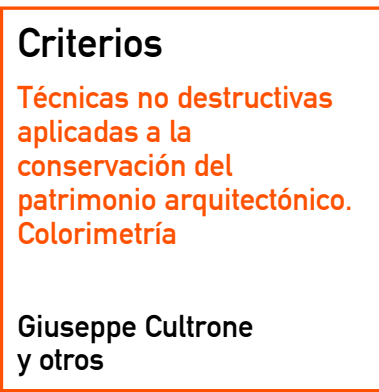

Se han preparado, además, nuevas probetas de ladrillos a las que se han adicionado cenizas volantes en diferentes proporciones. El objetivo es comprobar la cantidad máxima de este producto que se puede añadir sin que haya cambios sustanciales en el color de las piezas cocidas. Se han hecho ensayos de colorimetría con adiciones de 0,5, 1, 2, 5, 10 y $15 \%$ en peso de aditivo y la temperatura de cocción elegida ha sido de $900^{\circ} \mathrm{C}$. Las cenizas volantes proceden del electrofiltro de la central térmica "Los Barrios" de Sevilla. Su uso podría ser útil en el campo de la industria ladrillera ya que hoy en día se tiende a reutilizar diferentes productos de residuo para fabricar ladrillos de buena calidad (Domínguez y Ullmann, 1996; Wiebusch y Seyfried, 1997; Wiebusch et al., 1997 y 1998).

Estas cenizas son muy ricas en $\mathrm{SiO}_{2}$ y $\mathrm{Al}_{2} \mathrm{O}_{3}$ y están constituidas por partículas esféricas de color grisáceo cuyo tamaño oscila entre 0,5 y $100 \mu \mathrm{m}$ (foto 1).Para la medición del color se ha utilizado un colorimetro CR-210 portátil, cuyo sistema óptico usa una gran área de iluminación (foto 2).

El ángulo de visión es de $0^{\circ}$ para obtener lecturas que estén en correlación directa con el color tal como es visto bajo condiciones típicas de iluminación. Una lámpara de arco de xenón dentro de un compartimento mezclador proporciona difusión incluso alumbrando sobre un área de $50 \mathrm{~mm}$ de diámetro. Seis fotocélulas de silicio de alta sensibilidad miden luz incidente y luz reflejada. Las fotocélulas convierten la luz recibida por ellas en corriente cuya fuerza es proporcional a la luminosidad de la luz. Un microcomputador interno transforma estas señales en valores numéricos.

\section{Resultados}

Modificación del color en función de la temperatura de cocción

En la tabla 1 están representadas las medidas del color según los sistemas CIELab y CIECH para los dos tipos de arcilla cocidas a diferentes temperaturas.

Como es visible en la tabla 1 , las muestras no cocidas ( $V$ y $G$ ) son las más luminosas; sin embargo son grisáceas, especialmente por el valor tendente a cero del parámetro $a^{*}$. Destacan también las muestras de más alta temperatura de cochura, ya que el grado de vitrificación de la masa es elevado y se observan partes ennegrecidas que les confieren un color más oscuro. Esto ocurre especialmente en la muestra $\mathrm{G} 1100^{\circ} \mathrm{C}$, cuyo valor de luminosidad es el más bajo encontrado $\left(L^{*}=42,54\right)$.

Según la "Rock-Color-Chart" (Goddard et al., 1979) ambos grupos de ladrillos cocidos pueden definirse como rojo-amarillentos. Sin embargo, los de Guadix tienen valores más altos de $a^{*}$ y b*
Tabla 1. Valores de luminosidad $\left(L^{*}\right)$, cromaticidad $\left(a^{*} y b^{*}\right)$, color $\left(C^{*}\right)$ y ángulo de matiz $\left(\mathrm{H}^{\circ}\right)$ calculadas sobre las muestras de Viznar $(V)$ y Guadix $(G)$, crudas y cocidas entre 700 y $1100^{\circ} \mathrm{C}$

\begin{tabular}{|c|c|c|c|c|c|}
\hline Muestras & $L^{*}$ & $a^{*}$ & $b^{*}$ & $C^{*}$ & $\mathrm{H}^{\circ}$ \\
\hline V & 58,47 & 0,94 & 13,41 & 13,44 & $86^{\circ}$ \\
\hline V $700^{\circ} \mathrm{C}$ & 53,61 & 13,80 & 21,43 & 25,49 & $57^{\circ}$ \\
\hline $\mathrm{V} 800^{\circ} \mathrm{C}$ & 54,79 & 16,63 & 23,63 & 28,90 & $55^{\circ}$ \\
\hline V $900^{\circ} \mathrm{C}$ & 55,75 & 16,15 & 21,89 & 27,20 & $54^{\circ}$ \\
\hline V $1000^{\circ} \mathrm{C}$ & 57,80 & 15,01 & 21,59 & 26,30 & $55^{\circ}$ \\
\hline V $1100^{\circ} \mathrm{C}$ & 54,90 & 8,76 & 20,48 & 22,27 & $67^{\circ}$ \\
\hline G & 56,26 & 0,43 & 18,23 & 17,96 & $89^{\circ}$ \\
\hline G $700^{\circ} \mathrm{C}$ & 50,57 & 17,00 & 22,95 & 28,56 & $53^{\circ}$ \\
\hline G $800^{\circ} \mathrm{C}$ & 51,98 & 18,26 & 24,36 & 30,44 & $53^{\circ}$ \\
\hline G $900^{\circ} \mathrm{C}$ & 55,59 & 19,29 & 26,97 & 33,16 & $54^{\circ}$ \\
\hline G $1000^{\circ} \mathrm{C}$ & 54,20 & 19,80 & 25,35 & 32,17 & $52^{\circ}$ \\
\hline G $1100^{\circ} \mathrm{C}$ & 42,54 & 18,03 & 16,47 & 24,42 & $42^{\circ}$ \\
\hline
\end{tabular}

que los de Viznar, y esto se debe a la diferente composición de la arcilla de partida. En efecto, el principal elemento en la coloración de los ladrillos es la hematites que cristaliza después de la descomposición de los filosilicatos (Parras et al., 1996). Su contenido es más elevado en las muestras de Guadix que en las de Viznar. En este último grupo, a pesar de tener casi el mismo contenido en Fe tot que Guadix (Cultrone, 2001), el hierro presente queda "atrapado" en la red de los silicatos y aluminosilicatos de calcio inhibiendo la formación de hematites (Klaarenbeek, 1961; Kreimeyer, 1987; Maniatis et al., 1981).

Colores como rojo o pardo se obtienen por la presencia de pequeños granos de hematites dispersos en la matriz del ladrillo. Esta fase mineral es abundante y está presente en mayor o menor medida en todas las materias primas cerámicas a las que imparte la coloración pardo-rojiza. Pero existen otros minerales, como rutilo, cromita, arsénico o pirolusita, que también pueden determinar una variación en el color de las piezas cerámicas (Singer y Singer, 1963); asimismo, la temperatura y el ambiente oxidante o reductor de cocción influyen en el color (Manning, 1995; Cultrone y Sebastián, 2003). Algunos óxidos cromáticos, si se encuentran combinados en forma de silicatos, se descomponen por el calor, perdiendo oxigeno y cambiando de color; por enfriamiento, pueden absorber oxígeno y retornar a su estado y color originales.

\section{Modificación del color mediante el uso de cenizas volantes}

En lo que atañe a la modificación del color por adición de cenizas volantes, en todas las muestras se ha visto cómo, independientemente de la cantidad añadida, se desplazan los valores hacia el campo de los grises. En los gráficos 1 y 2 se observa un comportamiento diferente entre Víznar y Guadix, ya que en el segundo grupo de muestras se necesita al menos un $15 \%$ de ceni- 

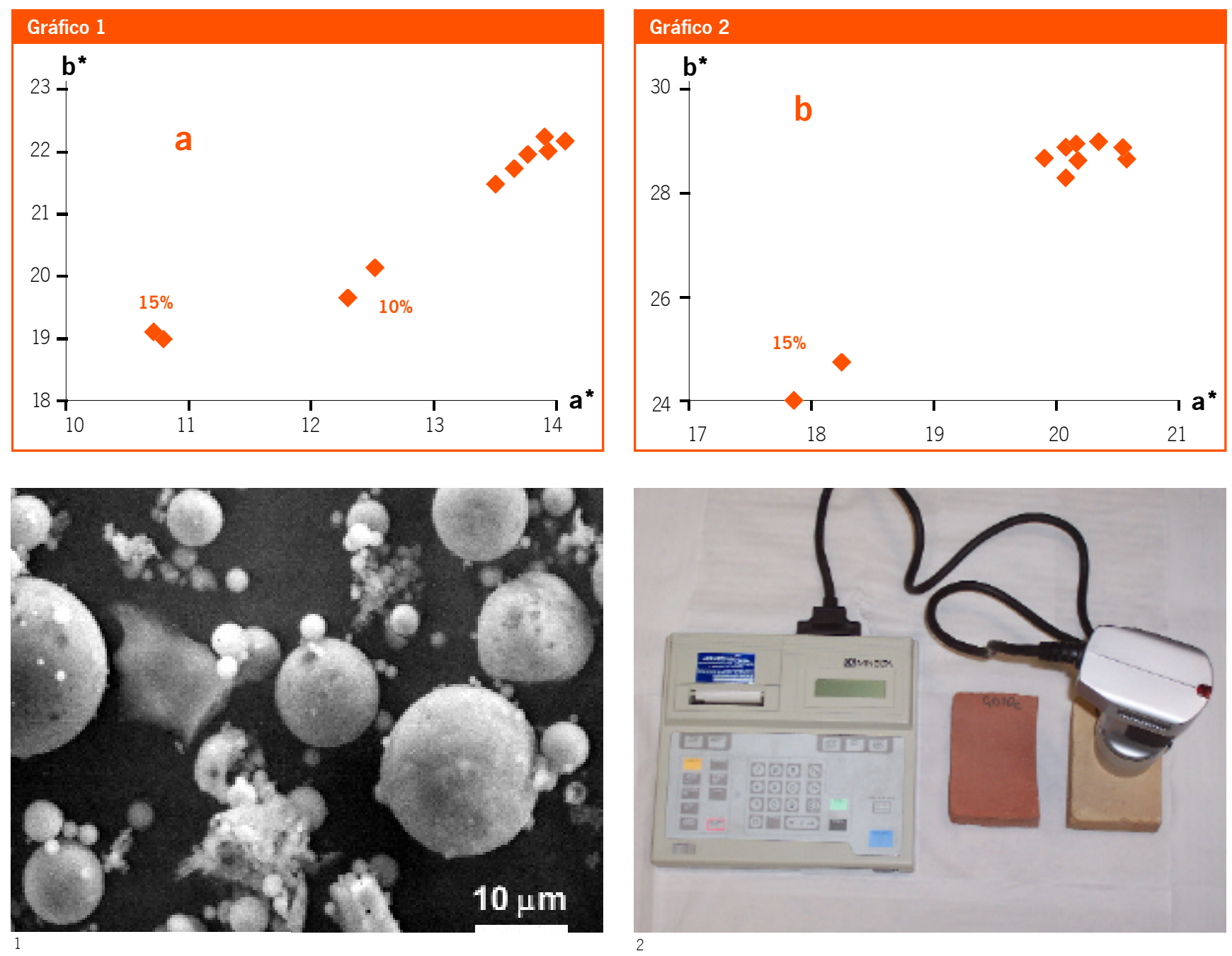

za volante para llegar a variar considerablemente su color, mientras que en Viznar, con sólo un 10\% se produce ese hecho en la mayoria de las muestras. Por tanto, una cantidad de ceniza volcánica inferior al $10 \%$ en peso es la adecuada a ser añadida a tierras arcillosas de composición parecidas a las de Viznar o Guadix si se quiere mantener homogénea la cromaticidad de los ladrillos con y sin aditivos.

\section{Conclusiones}

Se comprueba la utilidad y las ventajas que aporta la colorimetría a los estudios relacionados con la conservación del Patrimonio Arquitectónico.

Se ha notado una diferencia substancial en el color de dos grupos de ladrillos cocidos a temperaturas comprendidas entre 700

y $1100^{\circ} \mathrm{C}$. La presencia de calcita en la materia prima de Viznar hace que las muestras se vuelvan más amarillentas con respecto a las de Guadix, carentes de calcita y más ricas en hematites. Dentro del mismo grupo las diferencias no son tan marcadas y son apreciables sólo a nivel instrumental. Este hecho nos indica la utilidad del colorimetro. Se ha utilizado esta técnica también para medir la variación del color en función del porcentaje de un aditivo que se añade a la arcilla. En este caso, ladrillos que "de visu" parecen idénticos, la colorimetría permite asegurar que no es así. En concreto, se puede comparar el color de dos objetos y, de forma relativamente sencilla, cuantificar sus diferencias, hecho que es difícil de establecer con el ojo humano y sobre todo para objetos de tono similares.

Otras ventajas del colorímetro utilizado son su fácil manejo, su rapidez en las medidas, que siendo portátil, permite hacer medidas "en situ", y, principalmente, que es una técnica no destructiva. 


\section{Criterios}

Técnicas no destructivas aplicadas a la

conservación del patrimonio arquitectónico

Colorimetría

Giuseppe Cultrone

y otros

\section{Agradecimientos}

Este trabajo de investigación ha sido subvencionado por el proyecto DGI-MAT-2000-1457 y el grupo de investigación de la Junta de Andalucia RNM 179.

\section{Bibliografía}

CARRETA, A.; GIOVANNONE, C. (1989) Alterazione dei protettivi e fissativi sintetici applicati su supporti porosi interessati da fenomeni di migrazione e cristallizzazione di sali solubili: simulazioni sperimentali in laboratorio. $1^{\circ}$ Simposio Internazionale "La conservazione dei monumenti nel bacino del Mediterraneo", Bari, p.503-509

CULTRONE, G. (2001) Estudio mineralógico-petrográfico y físico-mecánico de ladrillos macizos para su aplicación en intervenciones del Patrimonio Histórico. Tesis Doctoral, Universidad de Granada, 267 p.

CULTRONE, G.; RODRÍGUEZ NAVARRO, C.; SEBAS.TIÁN, E.; CAZALLA, 0.; DE LA TORRE, M. J. (2001) Carbonate and silicate phase reactions during ceramic firing. European Journal of Mineralogy, 13, p. 621-634

CULTRONE, G.; SEBASTIÁN, E. (2003) Los materiales cerámicos en el Patrimonio Arquitectónico. Cuadernos Técnicos "Metodología de diagnóstico y evaluación de tratamientos para la conservación de los edificios históricos", 8, Cap. 2, p. 48-57

CULTRONE, G.; SEBASTIÁN, E.; CAZALLA, 0.; RODRÍGUEZ NAVARRO, C.; DE LA TORRE, M. J. (2002) Decay behaviour of bricks and its evaluation using colorimetry. En: Protection and Conservation of the Cultural Heritage of the Mediterranean Cities. Swets \& Zeitlinger, Lisse, p.145-152

DERBEZ, M.; LEFEVRE, R. A. (1996) Le contenu microparticulaire des croutes gypseuses de la cathédrale Saint-Gatien de Tours. Comparison avec l'air et la pluite. $8^{\text {th }}$ International Congress on Deterioration and Conservation of stone. Berlín, p. 359-370

DOMíNGUEZ, E. A.; ULLMANN, R. (1996) "Ecological bricks" made with clays and steel dust pollutants. Applied Clay Science, 11, p.237-249

DURÁN SUÁREZ, J. A. (1996) Estudio de consolidantes y protectivos para restauración de material pétreo. Tesis Doctoral, Universidad de Granada, 370 p.

ESBERT, R. M.; ORDAZ, J.; ALONSO, F. J.; MONTOTO, M.; GONZÁLEZ LIMÓN, T; ÁLVAREZ DE BUERGO BALLESTER, M. (1997) Manual de diagnosis y tratamiento de materiales pétreos y cerámicos. Collegi d'Apparelladors i Arquitectes Tècnics de Barcelona, Barcelona, 139 p.

FASSINA, V. (1976) A recent survey on air pollution in Venice in relation to the deterioration of marble and stone. Lithoclastia, 2, p. 33-43

GARCÍA PASCUA, N.; SÁNCHEZ DE ROJAS, M.; FRIAS, M. (1988) The important role of colour measurement in restoration Works. Use of consolidants and water repellents in sandstone. $8^{\text {th }}$ International Congress on deterioration of stone, Berlin, p. 1351-1361

GARCíA PASCUA, N.; SÁNCHEZ DE ROJAS, M.; FRÍAS, M. (1999) Medidas de color en diferentes materiales de construcción. La restauración en edificios de arenisca. Materiales de Construcción, 49, p. 29-45

GODDARD, E. N.; TRASK, P. D.; DE FORD, R. K; ROVE, O. N.; SINGEWALD, J. T.; QUERBECK, R. M. (1979) Rock Color Chart. The geological Society of America, Boulder, Co USA
KLAARENBEEK, F. W. (1961) The development of yellow colours in calcareous bricks. Transaction British Ceramic Society, 60, p. 738-772

KREIMEYER, R. (1987) Some notes on the firing colour of clay bricks. Applied Clay Science, 2, p. 175-183

MANIATIS, Y.; SIMOPOULOS, A.; KOSTIKAS, A. (1981) Moessbauer study of the effect of calcium content in iron oxide transformations in fired clays. Journal of American Ceramic Society, 64-5, p. 263-269

MANNING, D. A. C. (1995) Introduction to industrial minerals. Chapman \& Hall Ed., $276 \mathrm{p}$.

MINISTERIO DE OBRAS PÚBLICAS Y URBANISMO (1991) NBE FL-90: muros resistentes de fábrica de ladrillo. Madrid: MOPU, Secretaria General Técnica. Centro de Publicaciones, DL, 1991

MUNSELL COLOR (1994) Munsell Soil Color Charts. Baltimore, Maryland. Revised edition

PALENI, A.; CURRI, S. B. (1976) La contaminazione biologica sulla superficie delle opere d'arte. Proceeding of the International Symposium "The Conservation of Stone", Bologna, p. 281-293

PARRAS, J.; SÁNCHEZ JIMÉNEZ, C.; RODAS, M.; LUQUE, F. J. (1996) Ceramic applications of middle Ordovician shales from central Spain. Applied Clay Science, 11, p. 25-41

RODRÍGUEZ NAVARRO, C.; CULTRONE, G.; SÁNCHEZ NAVAS, A.; SEBASTIÁN, E. (2003) TEM study of mullite growth alter muscovite breakdown. American Mineralogist, 88, p. 713-724

RODRÍGUEZ NAVARRO, C.; SEBASTIÁN PARDO, E. (1994) Pollution-derived heavy metal enrichment on decayed building stones. Mineralogical Magazine, 59A, p. 781-782

RODRÍGUEZ NAVARRO, C.; SEBASTIÁN PARDO, E. (1996) Role of particulate matter from vehicle exhaust on porous building stones (limestone) sulfation. Science of the Total Environment, 187, p. 79-91

SEBASTIÁN, E.; ZEZZA, U. (1998) Colour parameters of calcarenites used in the architectural heritage of Andalusia (Spain) and Apulia-Basilicata (Italy). IV Congreso Internacional de Rehabilitación del Patrimonio Arquitectónico y Edificación. La Habana (Cuba), p. 560-572

SINGER, S.S.; SINGER,F. (1963). Industrial ceramics. Londres: Chapman and Hall, 1963

WARSCHEID, T.; BRAAMS, J. (2000) Biodeterioration of stone: a review. International Biodeterioration \& Biodegradation, 46, p. 343-368

WIEBUSCH, B.; HAUCH, D.; SEYFRIED, C. F. (1997) Impact of sewage sludge ashes on brick manufacturing. Technical aspects and environmental compatibility. $7^{\text {th }}$ Japanese-German Workshop on Waste and Sludge Treatment, Kyoto, p. $1-14$

WIEBUSCH, B.; OZAKI, M.; WANTANABE, H.; SEYFRIED, C. F. (1998) Assessment of leaching tests on construction material made of incinerator ash (sewage sludge): investigations in Japan and Germany. Water Science Technology, 38, p. 195-205

WYSZECKI, G., AND STILES, W.S. (1982) Color Science: Concepts and Methods, Quantitative Data and Formulae. Nueva York: John Wiley and Sons, 1982 (Segunda Edición) 